\title{
Pensamento Computacional: Um estudo empírico sobre as questões de matemática do PISA
}

\author{
Palloma A. A. Mestre ${ }^{12}$, Wilkerson L. Andrade ${ }^{1}$, Dalton S. Guerrero ${ }^{1}$, \\ Lívia Sampaio $^{1}$, Rivanilson da Silva Rodrigues ${ }^{1}$ \\ Erick John Fidelis Costa ${ }^{1}$, \\ ${ }^{1}$ Laboratório de Práticas de Software - SPLab. \\ Universidade Federal de Campina Grande (UFCG) \\ Campina Grande - PB - Brasil \\ ${ }^{2}$ Coordenação de Tecnologia da Informação e Comunicação - CTIC. \\ Universidade Estadual da Paraíba (UEPB) \\ Campina Grande - PB - Brasil \\ \{palloma.alencar, erick.costa.ti, rivanilson.sb\}@gmail.com \\ \{wilkerson, livia, dalton\}@computacao.ufcg.edu.br
}

\begin{abstract}
The proposal of Computational Thinking is to provide a method to solve problems in various knowledge areas, using computational concepts. However, in the literature there are few studies that evaluate the relationship of Computational Thinking and areas such as mathematics. This paper presents an empirical investigation with aim of assessing whether there is a relationship between the Computational thinking and the PISA math problems. The results indicate that there is a relationship between the evaluated skills, and that the nine concepts of computational thinking used in this study six are present in the set of studied questions.
\end{abstract}

Resumo. A proposta do Pensamento Computacional é fornecer um método para solucionar problemas nas diversas áreas do conhecimento, usando conceitos computacionais. No entanto, na literatura existem poucos estudos que avaliem a relação do Pensamento Computacional e áreas como a Matemática. O presente trabalho apresenta uma investigação empírica com intuito de avaliar se há relação entre o Pensamento Computacional e os problemas de matemática do PISA. Os resultados indicam que existe relação entre as habilidades avaliadas, e que dos nove conceitos do Pensamento Computacional utilizados neste estudo seis estão presentes no conjunto de questões estudadas.

\section{Introdução}

O Pensamento Computacional (PC) contribui para o processo de resolução de problemas nos diversos contextos da sociedade, permitindo que os indivíduos possam aplicar a computação nas suas ações cotidianas [Gomes and Melo 2013]. De acordo com [Wing 2006], PC é um método utilizado para solucionar problemas, conceber sistemas e compreender o comportamento humano inspirado em conceitos fundamentais da Ciência da Computação. Na prática, esses conceitos podem ser aplicados transversalmente ao conteúdo de disciplinas, tanto da educação básica (e.g. Matemática, Ciências, Artes, etc.) 


\section{CBIE-LACLO 2015}

Anais dos Workshops do IV Congresso Brasileiro de Informática na Educação (CBIE 2015)

como da educação superior (e.g. Psicologia Educacional, Estudos Liberais, etc.) por meio de técnicas de resolução de problemas [Yadav et al. 2014].

Vários países adotaram estratégias para inserção do PC na educação básica, entre eles estão Estados Unidos, Reino Unido, Israel, Alemanha, Holanda, Noruega, Nova Zelândia e Dinamarca [Caspersen and Nowack 2013]. Ao observar estas estratégias, percebe-se que as abordagens propostas consideram o contexto ao qual o PC será inserido. Geralmente as definições que norteiam estas estratégias são similares, mas adaptações no conteúdo didático, métodos pedagógicos, atividades desenvolvidas e ferramentas utilizadas são necessárias para viabilizar a sua efetiva implantação. Um exemplo destas dificuldades está descrito no trabalho de [de Souza et al. 2014], em que os autores ilustram as barreiras encontradas para aplicação do PC, em escolas brasileiras, através de um projeto de jogos americano. Os maiores obstáculos encontrados foram as diversidades culturais, socioeconômicas e a formação insuficiente dos professores brasileiros na área de PC.

Com o objetivo de promover uma discussão acerca das habilidades estimuladas pelo PC e as Capacidades Fundamentais da Matemática, no contexto da resolução de problemas, necessárias para que os alunos adquiram o letramento em matemática, foi conduzido um estudo empírico. Assim, espera-se ampliar a compreensão de como as habilidades do PC se relacionam com as Capacidades da Matemática. Além disso, fazse necessário verificar se as habilidades abordadas nos problemas de matemática estão relacionadas ao PC. Para esta pesquisa foram considerados os parâmetros requeridos no nível de letramento em matemática definido pelo Programa Internacional de Avaliação do Aluno - PISA. A escolha do PISA foi motivada pelo fato do seu letramento em matemática avaliar a capacidade do aluno em formular, aplicar e interpretar a matemática em diferentes contextos [OCDE 2014]. Além disso, apesar dos avanços da educação brasileira nos últimos anos, os resultados do PISA 2012 apresentam um baixo desempenho dos alunos brasileiros na disciplina de matemática em relação aos demais países participantes (posição 58 do total de 65).

Neste estudo foram avaliadas todas as questões de matemática do PISA (total de 161 questões) publicadas pela $\mathrm{OCDE}^{1}$ entre os anos de 2000 e 2012. O intuito é verificar se as habilidades que os alunos precisam para resolver estas questões são similares às habilidades estimuladas pelo PC. Em caso afirmativo, apresentar a relação entre estas habilidades por meio de um comparativo.

Os resultados alcançados apontam que o PC está amplamente relacionado com a resolução de problemas. Além disso, observou-se que as habilidades estimuladas pelo PC são similares às habilidades abordadas nos problemas de matemática de avaliações baseadas em resolução de problema, como o PISA.

Este artigo está divido em 6 seções. Na Seção 2 tem-se uma breve revisão da literatura. Na Seção 3 são apresentados alguns trabalhos relacionados. Já na Seção 4 descreve-se o estudo empírico, apresentando a metodologia utilizada. Na Seção 5 os resultados alcançados e a discussão são abordadas. Por fim, na Seção 6 são expostas as considerações finais e propostas para trabalhos futuros.

\footnotetext{
${ }^{1}$ A OCDE disponibiliza apenas uma amostra das questões aplicadas.
} 
CBIE-LACLO 2015

Anais dos Workshops do IV Congresso Brasileiro de Informática na Educação (CBIE 2015)

\section{Pensamento Computacional}

Conforme mencionado anteriormente, PC é um método utilizado para resolver problemas inspirado nos conceitos da Ciência da Computação. É importante ressaltar que esses conceitos não são exclusivos da Ciência da Computação, no entanto, a proposta do PC é abordar esses conceitos sobre uma perspectiva computacional, envolvendo, em alguns casos, ferramentas tecnológicas.

A Computer Science Teachers Association (CSTA) e International Society for Technology in Education (ISTE) elaboraram uma abordagem para aplicação do PC na educação básica americana (K-12), por meio da criação e disponibilização de um conjunto de materiais, contendo definições operacionais, vocabulários, experiências de aprendizagem, e cenários onde o PC pode ser utilizado em sala de aula (Computational Thinking Teacher Resources), com objetivo de inspirar os professores que não são da área da Ciência da Computação [CSTA\&ISTE 2011].

Em sua definição operacional o CSTA e ISTE propuseram um conjunto de conceitos e capacidades do PC. Esses conceitos envolvem habilidades como: (i) coleta de dados: o processo de coletar informações de forma adequada; (ii) análise de dados: dar sentido aos dados, encontrar padrões e tirar conclusões; (iii) representação de dados: representar e organizar os dados em gráficos, tabelas, textos e imagens; (iv) decomposição de problemas: quebrar tarefas em partes gerenciáveis, menores; (v) abstração: reduzir a complexidade para definir a ideia principal; (vi) algoritmo e procedimentos: definir um conjunto de passos ordenados para resolver um problema ou atingir algum fim; (vii) automação: usar os computadores ou máquinas para fazer tarefas repetitivas e tediosas; (viii) paralelização: organizar recursos para, simultaneamente, realizar tarefas para alcançar um objetivo comum; (ix) simulação: representar ou modelar um processo.

As habilidades estimuladas pelo PC estão diretamente relacionadas à resolução de problemas. Essas habilidades envolvem a capacidade de ler, interpretar textos, bem como, compreender as situações reais propostas nos problemas e transpor as informações destas situações para modelos matemáticos, científicos ou sociais.

No contexto da matemática, a resolução de problemas tem uma contribuição significativa [Wilson et al. 1993]. Os alunos precisam desenvolver habilidades que vão além dos cálculos, eles precisam ter a capacidade de descrever, explicar ou prever fenômenos, utilizando várias formas de raciocínio [OCDE 2014]. O PISA no seu nível de letramento em matemática, descreve um conjunto de capacidades fundamentais que estão relacionadas com o processo matemático [OCDE 2014]. Estas capacidades envolvem: (i) a comunicação através do processo de leitura, interpretação e decodificação das situaçõesproblemas; (ii) a matematização que é a transposição de um problema do mundo real para uma forma matemática; (iii) a representação por meio da seleção, interpretação, utilização e tradução de representações para capturar as situações-problema ou interagir com a resposta; (iv) o raciocínio e a argumentação que envolve o pensamento lógico e análise de elementos do problema de modo a permitir inferências ou fornecer justificativas para solução do problema; (v) o delineamento de estratégias para resolução de problemas por meio de processos de controle para reconhecimento, formulação e resolução de problemas; (vi) a utilização de linguagem simbólica, formal e técnica e de operações envolve a compreensão, interpretação, manipulação e utilização de expressões, símbolos dentro do contexto da matemática; e (vii) a utilização de ferramentas matemáticas que compreende 


\section{CBIE-LACLO 2015}

Anais dos Workshops do IV Congresso Brasileiro de Informática na Educação (CBIE 2015)

o conhecimento e aptidão para lidar com ferramentas matemáticas.

\section{Trabalhos Relacionados}

Apesar dos estudos acerca do PC não serem recentes, percebe-se que ainda não existe um consenso de como e quando se deve introduzi-lo na educação. Para [Resnick 2007] os alunos da educação básica, i.e., o ensino primário e secundário, devem ser submetidos ao processo de projetar, criar, experimentar e explorar aperfeiçoando suas habilidades criativas, assim como ocorre no jardim da infância. $\mathrm{O}$ autor ressalta que a utilização de ferramentas de programação visual e de encaixe (e.g., Scratch) auxiliam no processo criativo e sugere a adoção do ensino de programação nos diversos anos da educação básica em disciplinas complementares. Ainda neste contexto, metodologias para viabilizar o ensino do PC por meio do ensino de programação utilizando ferramentas como o Scratch estão disponíveis na literatura [de França and do Amaral 2013], [von Wangenheim et al. 2014]. Nestes trabalhos os autores propõem um conjunto de atividades, por meio da programação, para ensinar os conceitos computacionais. Em alguns casos, essas atividades abordam conteúdos de disciplinas da educação formal (e.g, matemática, ciências, português, artes e etc.).

Outra abordagem é o ensino do PC mediante a aplicação de atividades lúdicas. No trabalho de [Andrade et al. 2013] são apresentadas três atividades lúdicas desenvolvidas, para aplicação no ensino fundamental, de forma a favorecer a disseminação do PC. Neste trabalho os autores introduzem os conceitos do PC proposto pelo [CSTA\&ISTE 2011] sem uso do computador.

O trabalho apresentado em [Barcelos and Silveira 2012], assim como este trabalho, propõe uma associação das competências da matemática com as competências do PC. No entanto, Barcelos e Silveira abordam as competências da matemática previstas nos Parâmetros Curriculares Brasileiros, relacionando as atividades didáticas do PC com a matemática. O presente trabalho difere da proposta de Barcelos e Silveira em três aspectos: (i) a análise realizada envolve as Capacidades Fundamentais da Matemática definidas no nível de letramento do PISA e os conceitos do PC; (ii) no estudo de caso foram avaliadas questões típicas da matemática, enquanto que Barcelos e Silveira apresentam seus resultados no contexto da criação de algoritmos e/ou programas; (iii) o intuito deste estudo é apresentar uma discussão acerca da associação das habilidades estimuladas pelo PC e a matemática. Já Barcelos e Silveira abordam uma discussão entre a relação do ensino de Computação e suas possíveis relações com a disciplina da Matemática.

\section{Um Estudo Empírico sobre o PISA}

O objetivo deste estudo empírico é investigar, através de um estudo de caso, se há indícios de que as habilidades abordadas nos problemas de matemática estão relacionadas às habilidades estimuladas pelo PC. Considera-se uma relação válida, se um ou mais conceitos de PC foram cobertos pelo conjunto de questões avaliadas. Outro objetivo deste estudo, é apresentar como as habilidades estimuladas pelo PC podem ser associadas às Capacidades Fundamentais da Matemática previstas no nível de letramento do PISA. Para atingir o objetivo proposto, o estudo foi dividido em três etapas: (i) revisão da literatura acerca das definições e abordagens do PC; (ii) classificação das questões de matemática do PISA considerando os conceitos do PC; (iii) associação entre os conceitos do PC e as Capacidades Fundamentais da Matemática. 


\section{CBIE-LACLO 2015}

Anais dos Workshops do IV Congresso Brasileiro de Informática na Educação (CBIE 2015)

Na primeira etapa deste estudo foi realizada uma revisão da literatura com intuito de identificar definições do PC que pudessem ser aplicadas no contexto da educação formal. Para isso, foram considerados os conceitos apresentados pelo [CSTA\&ISTE 2011]: Coleta de Dados, Análise de Dados, Representação de Dados, Decomposição de Problemas, Abstração, Algoritmo e Procedimentos, Automação, Paralelização e Simulação.

$\mathrm{Na}$ segunda etapa, todas as questões liberadas pela OCDE (161), referentes às provas de matemática do PISA aplicadas nos anos entre 2000 e 2012 foram classificadas de acordo com a presença ou não de conceitos de PC propostos pelo [CSTA\&ISTE 2011]. Cada questão foi classificada manualmente por três pesquisadores da área de PC do nosso grupo de pesquisa de forma que uma votação majoritária permitiu indicar se um conceito estava ou não presente na questão a ser classificada. É importante salientar que antes da classificação das questões, foi realizado um treinamento com todos os participantes, onde os conceitos de PC foram discutidos no contexto da matemática. Além disso, a avaliação de cada questão ocorreu de forma independente.

Por fim, todos os conceitos de PC identificados nas questões foram associados às Capacidades Fundamentais da Matemática observando três aspectos: objetivos de aprendizagem de cada questão; capacidades matemáticas avaliadas pelo PISA e as características dos conceitos do PC. O objetivo desta etapa é fornecer resultados preliminares acerca da viabilidade da associação dos conceitos do PC à disciplina de matemática.

\section{Resultados e Discussão}

Dos nove conceitos de PC considerados para a classificação das questões de matemática do PISA, seis foram identificados em pelo menos uma questão. Esses conceitos são: Abstração, Análise de Dados, Decomposição de Problemas, Representação de Dados, Coleta de Dados e Algoritmos e Procedimentos. A Seção 5.1 apresenta os resultados quantitativos da relação entre os problemas de matemática e o PC. Já a Seção 5.2 mostra como podemos associar as habilidades estimuladas pelos conceitos de PC às Capacidades Fundamentais da Matemática definidas pelo PISA.

\subsection{Relação entre os Problemas de Matemática e o Pensamento Computacional}

Analisando o gráfico da Figura 1 percebe-se que os conceitos de Abstração e Análise de Dados foram cobertos em 100\% e 98,76\% das questões, respectivamente. Estes conceitos na computação envolvem a capacidade de reduzir a complexidade de um problema e identificar as situações principais como variáveis, expressões e etc. Além disso, envolve a habilidade de interpretar problemas e suas soluções. No contexto do PISA, os conceitos de Abstração e Análise de Dados estão relacionados a competência de ler, decodificar e interpretar as afirmações, perguntas e tarefas, afim de, transpor um problema no mundo real para uma forma estritamente matemática, sendo capaz de, não só, encontrar a solução do problema mas de explicar e/ou justificar as soluções propostas.

Ao avaliarmos as questões, percebe-se que o foco do PISA não é que o aluno resolva cálculos complexos ou problemas longos, mas que tenha capacidade de empregar a matemática na resolução de problemas do cotidiano. Essa característica pode ter influenciado os resultados no que diz respeito aos conceitos Algoritmos e Procedimentos e Coleta de Dados, que obtiveram menor cobertura. 
CBIE-LACLO 2015

Anais dos Workshops do IV Congresso Brasileiro de Informática na Educação (CBIE 2015)

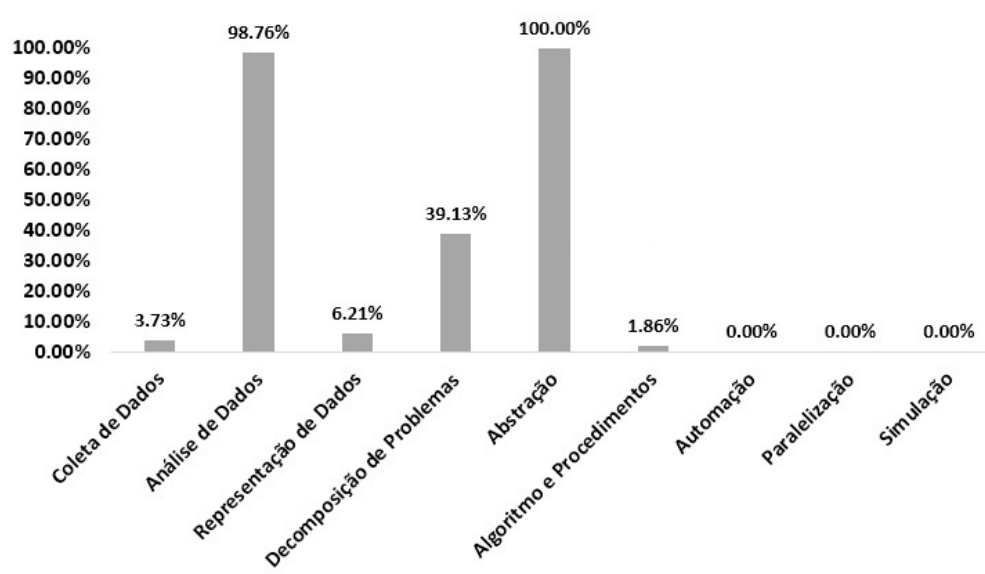

Figura 1. Distribuição dos Conceitos do Pensamento Computacional identificados nas questões do PISA

Os conceitos Automação, Paralelização e Simulação não foram cobertos em nenhuma questão avaliada. Estes conceitos, em geral, estão relacionados ao uso de ferramentas matemáticas, como: computadores, jogos, robôs. Neste estudo, foram avaliadas atividades típicas da matemática onde os alunos não utilizaram ferramentas matemáticas para auxiliar no processo de resolução.

Apesar dos 9 conceitos de PC não estarem cobertos pelas questões do PISA, temse indícios de que as habilidades estimuladas pelo PC estão relacionadas as habilidades necessárias para resolver as questões do PISA uma vez que, pelo menos, seis foram identificados. As habilidades estimuladas pelos conceitos do PC contribuem para o desenvolvimento do pensamento algorítmico, lógico e sistemático [Curzon 2013], fatores importantes para o processo de resolução de problemas. Por meio destes dados preliminares, observa-se que caso os alunos sejam treinados na resolução de questões de matemática que envolvam os Conceitos do PC, estes possivelmente terão êxito em avaliações como o PISA e em avaliações semelhantes (por exemplo, o Exame Nacional do Ensino Médio ENEM).

\subsection{Associação entre as Capacidades Fundamentais da Matemática e as Habilidades Estimuladas pelo Pensamento Computacional}

Os conceitos do PC estão presentes nas questões de matemática avaliadas, conforme mostram os dados apresentados na Seção 5.1. Neste caso, temos indícios de que há uma relação entre o PC e problemas da matemática, pelo menos no contexto do PISA. Por isso, é importante traçar como os conceitos do PC podem ser associados, na prática, às Capacidades Fundamentais da Matemática sobre vários aspectos.

O conceito Abstração está relacionado com a compreensão das situaçõesproblemas. Os alunos devem ser capazes de entender estas situações identificando as ideias principais [CSTA\&ISTE 2011]. Na computação, a abstração é amplamente utilizada por meio do reuso de software ou parte dele. No contexto da matemática, a abstração envolve a capacidade de interpretar um problema proposto e, extrair suas premissas, variáveis e restrições. Por meio da abstração o aluno exercita a sua capacidade de leitura e interpretação de textos. Além disso, o aluno é motivado a delinear estratégias ou 


\section{CBIE-LACLO 2015}

Anais dos Workshops do IV Congresso Brasileiro de Informática na Educação (CBIE 2015)

selecionar as já existentes, utilizando a matemática na solução de problemas do mundo real, usando a linguagem formal, técnica e/ou simbólica.

O processo de análise de dados envolve a habilidade de compreender os dados, que foram obtidos por meio da coleta de dados, encontrando padrões e tirando conclusões. Na matemática a análise de dados está relacionada com a capacidade de extrair valores de gráficos, tabelas, listas, figuras e textos, com intuito de inferir, concluir fatos ou estimar novos valores [CSTA\&ISTE 2011]. Por meio da análise de dados espera-se que os alunos possam estruturar, conceituar, fazer suposições e/ou justificar a solução de um problema. Além disso, o pensamento lógico é envolvido mediante o processo de interpretação e avaliação dos resultados matemáticos. Em relação às capacidades fundamentais da matemática abordadas pelo PISA, a análise de dados estimula a comunicação, "matematização" e o raciocínio e argumentação.

A decomposição de problemas envolve a fragmentação de tarefas em partes gerenciáveis. Na matemática, esse conceito está relacionado com a execução dos cálculos. A decomposição de problemas é o processo de identificar e utilizar fórmulas, funções e procedimentos matemáticos necessários para solucionar o problema. Apesar da decomposição de problemas está relacionada, na maioria dos casos, à execução dos cálculos, este conceito contribui para o desenvolvimento de várias habilidades matemáticas no contexto da resolução de problemas. Os alunos ao utilizarem a decomposição devem ser capazes de transpor um problema definido no mundo real para uma forma ou modelo matemático, ampliando a sua capacidade de abstração. Além disso, esse conceito favorece a compreensão, interpretação, manipulação e utilização de expressões simbólicas dentro do contexto matemática.

Ao se deparar com os problemas matemáticos, geralmente os alunos estão preocupados em efetuar os cálculos corretos para encontrar a solução do problema. No entanto, a forma como essa solução será representada é tão importante quantos os cálculos. Portanto, a representação de dados envolve a capacidade para representar e organizar os dados, sejam em gráficos, tabelas, textos ou imagens. O PISA define como uma capacidade fundamental da matemática a representação de dados. Mediante a representação de dados os alunos podem selecionar, interpretar, traduzir e utilizar representações para interagir com o problema, além de, apresentar as suas soluções [CSTA\&ISTE 2011].

A coleta de dados envolve a capacidade de sistematizar métodos, de forma adequada, para obter informações, sejam de pessoas ou outras fontes de dados, tais como: arquivos, sistemas, formulários etc. Estas informações devem ser relevantes para solução ou compreensão do problema a ser resolvido. No contexto da matemática, a coleta de dados está relacionada ao processo de identificação dos dados quantitativos e qualitativos que são relevantes para resolução do problema [CSTA\&ISTE 2011]. Ao serem submetidos ao processo de coleta de dados os alunos desenvolvem aptidões para ler e interpretar as afirmações, perguntas, e objetos de um problema, contribuindo para o desenvolvimento da sua capacidade de comunicação, atendendo a expectativa do PISA.

Entende-se por algoritmos e procedimentos um conjunto de passos ordenados tomados para resolver um problema ou atingir algum fim. Na computação, este conceito é amplamente utilizado na resolução de problemas computacionais específicos mediante a transformação de entradas em saídas. Na matemática, os algoritmos e procedimen- 


\section{CBIE-LACLO 2015}

Anais dos Workshops do IV Congresso Brasileiro de Informática na Educação (CBIE 2015)

tos contribuem para sistematização das soluções dos problemas, por meio de uma série de passos bem definidos que devem ser seguidos para alcançar a solução desejada. De acordo com o nível de letramento em matemática definido pelo PISA [OCDE 2014] os alunos ao terminarem o ensino fundamental devem ser capazes de delinear ou utilizar algoritmos com os conceitos matemáticos. A utilização de algoritmos e procedimentos estimula a leitura, interpretação de textos e decodificação de situações-problemas para modelos matemáticos.

\section{Considerações Finais}

Os resultados do PISA 2012 apresentam os avanços da educação brasileira em relação à disciplina de matemática. O Brasil foi o país com o maior aumento de pontos em relação às edições passadas (334 pontos em 2000, 356 pontos em 2003, 370 pontos em 2006, 386 em 2009 e 391 pontos em 2012) [OCDE 2014]. Apesar destes avanços, o Brasil encontra-se na posição $58^{\circ}$ do ranking da OCDE (total de 65 países) nesta disciplina. Portanto, visando contribuir para melhoria deste cenário, esta pesquisa oferece um debate acerca do uso de PC na educação básica no contexto das avaliações. Um estudo inicial acerca de uma investigação sobre o relacionamento entre as questões de matemática do PISA e os conceitos do PC propostos por [CSTA\&ISTE 2011] foi apresentado. Após a análise dos resultados, foi identificado que: (i) dos nove conceitos do PC avaliados, seis foram identificados em pelo menos uma questão; (ii) os conceitos Abstração, Análise de Dados, Decomposição de Problemas e Representação de Dados são respectivamente, os conceitos mais frequentes nas questões enquanto que; (iii) os conceitos de Coleta de Dados e Algoritmo e Procedimento foram identificados em menos de 5\% das questões.

De acordo com a amostra avaliada, observa-se que apesar dos nove conceitos de PC não serem cobertos pelas questões, as habilidades estimuladas pelos conceitos do PC estão relacionadas com as Capacidades Fundamentais da Matemática. Portanto, há indícios de que a inserção do PC na educação básica, em disciplinas com a matemática, poderá auxiliar no processo de ensino-aprendizagem baseado em resolução de problemas, estimulando os alunos a utilizarem o raciocínio matemático em ambientes contextualizados.

Em continuidade à pesquisa, estão sendo realizados estudos acerca da criação de um framework para classificação de problemas matemáticos em relação ao PC. Através deste framework, professores de matemática poderão avaliar as suas atividades, bem como, obter problemas matemáticos através de um sistema Web que estejam relacionados ao PC, facilitando a inserção de exercícios que envolvam o uso de métodos de resolução de problemas, o que pode contribuir para que os alunos alcancem resultados cada vez melhores futuramente.

\section{Referências}

Andrade, D., Carvalho, T., Silveira, J., Cavalheiro, S., Foss, L., Fleischmann, A. M., Aguiar, M., and Reiser, R. (2013). Proposta de atividades para o desenvolvimento do pensamento computacional no ensino fundamental. In Anais do WIE, volume 1.

Barcelos, T. S. and Silveira, I. F. (2012). Pensamento computacional e educação matemática: Relações para o ensino de computação na educação básica. In XX WEI. 


\section{CBIE-LACLO 2015}

Anais dos Workshops do IV Congresso Brasileiro de Informática na Educação (CBIE 2015)

Caspersen, M. E. and Nowack, P. (2013). Computational thinking and practice: A generic approach to computing in danish high schools. In Proc. of the Fifteenth Australasian Computing Education Conference-Volume 136, pages 137-143. Australian Computer Society, Inc.

CSTA\&ISTE (2011). Computational thinking teacher resources. http://csta.acm.org/curriculum/sub/compthinking.html acesso: março/2015.

Curzon, P. (2013). cs4fn and computational thinking unplugged. In Proceedings of the 8th Workshop in Primary and Secondary Computing Education, pages 47-50. ACM.

de França, R. S. and do Amaral, H. J. C. (2013). Proposta metodológica de ensino e avaliação para o desenvolvimento do pensamento computacional com o uso do scratch. In Anais do WIE, volume 1.

de Souza, C. S., Salgado, L. C., Leitão, C. F., and Serra, M. M. (2014). Cultural appropriation of computational thinking acquisition research: Seeding fields of diversity. In ITiCSE '14, pages 117-122, New York, NY, USA. ACM.

Gomes, T. and Melo, J. (2013). O pensamento computacional no ensino médio: Uma abordagem blended-learning. In Anais do $21^{\circ}$ WEI-XXXIII CSBC. Maceió, AL-Brasil.

OCDE (2014). Relatório nacional pisa 2012 resultados brasileiros. http://portal.inep.gov.br/internacional-novo-pisa-resultados acesso: abril/2015.

Resnick, M. (2007). All i really need to know (about creative thinking) i learned (by studying how children learn) in kindergarten. In Proceedings of the 6th ACM SIGCHI Conference on Creativity \&Amp; Cognition, C\&C '07, pages 1-6, New York, NY, USA. ACM.

von Wangenheim, C. G., Nunes, V. R., and dos Santos, G. D. (2014). Ensino de computação com scratch no ensino fundamental-um estudo de caso. RBIE, 22(03):115.

Wilson, J. W., Fernandez, M. L., and Hadaway, N. (1993). Mathematical problem solving. Research ideas for the classroom: High school mathematics, pages 57-78.

Wing, J. M. (2006). Computational thinking. Communications of the ACM, 49(3):33-35.

Yadav, A., Mayfield, C., Zhou, N., Hambrusch, S., and Korb, J. T. (2014). Computational thinking in elementary and secondary teacher education. ACM Transactions on Computing Education (TOCE), 14(1):5. 